\title{
NEUROSCIENCE
}

\section{Sharing the light: an open database to help optimize optogenetics in nonhuman primates}

Tremblay, S. et al. Neuron 108, 1075-1090 (2020)

There's a lot of hype about optogenetics. The ability to manipulate and modulate neuronal activity with a little light holds therapeutic potential and has seen a few early moves into clinical trials for a number of neurological-related disorders, such as retinal degeneration that leads to blindness. But, there is a lot still to learn about applying the technique to primates.

"The technique has been optimized for a very small brain," says Sebastien Tremblay, a postdoctoral fellow in Michael Platt's lab at the University of Pennsylvania. That presents hurdles for applying optogenetics to larger animals, including humans.

Mice, the lab animals in which most optogenetic work has taken place over the past decade, have brains of about a cubic centimeter - considerably smaller than that of a macaque, let alone a human. The optic fibers used can illuminate about a millimeter of brain tissue. "Good enough for mice, not good enough for primates," Tremblay says. And whereas mice can be genetically engineered to express the necessary light-sensitive opsin proteins in their neurons, monkeys cannot (....at least not yet, though there have been some efforts in rat-sized marmosets). Nor will we be making genetically modified humans. "We have to innovate around that," he says.

Alternatively, viral vectors can deliver the opsins, but these still only make it to relatively small areas of the brain. For optogenetics to be effective in larger animals, larger areas of the primate brain need to be made sufficiently sensitive to light to be manipulated.

These are technical limitations, but they lead to a major problem: "It's really hard to get behavioral effects from optogenetics in primates," Tremblay says, the outcome the field needs to make sense of what is going on at the neuronal level. A number of labs working with nonhuman primates have taken on the challenge, but for every successfully manipulated animal that makes it into the published literature, how many are going unreported?

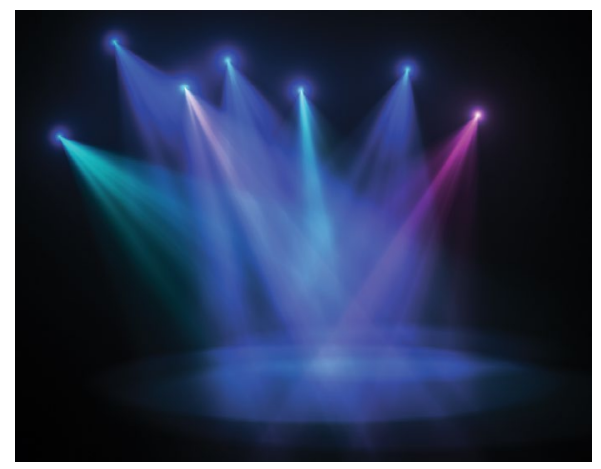

Light can manipulate neurons, if it can reach them. Credit: fotograzia / Getty

As he was planning his own optogenetic experiments, Tremblay realized details were hard to come by. "I thought, it's hard for me make informed decisions about my own experiments if I don't have access to all the information that's out there - both positive and negative results," he says. "Knowing what has been done is a very hard question in science."

Encouraged by the data sharing efforts of the nonhuman primate neuroimaging community and picking up on some initial work underway within MIT, he started asking around. Hundreds of emails to researchers became thousands, and the NHP Optogenetics Open Database came to be. Writing in Neuron, Tremblay and his colleagues describe an initial analysis of survey answers, which touch on the species, viral vectors, promotors, opsins, and reporters that have been used in different labs, along with physiological, anatomical, and behavior outcomes and success rates. The respondents - from 45 labs at around the world at the time of analysis - weren't stingy with their negative results either: 32 of them provided over 500 entries with unpublished results.

The data capture how different labs have approached applying optogenetic techniques to their primates, and where they struggle.
"I think the most important result that comes out is a confirmation that behavior is the challenge," Tremblay says. Many have had success in getting different opsins into the primate brain and have been able to see neurons that react to light, he says "but very few labs have managed to get strong, reliable behavioral results out of optogenetic stimulation in primates."

He notes that the field needs to be cautious about interpreting these preliminary results; they were limited to one parameter at a time, but different factors - say, species vs. viral vector - are likely to interact. The database is publically available and includes anonymized answers, and Tremblay hopes researchers continue to contribute their results so that more sophisticated analyses can be run to figure out what works - and what doesn't. "We really want to make sure that we limit the number of animals that are required to optimize this technique," he says, an ethical concern that goes hand in hand with striving for the best experimental outcomes.

As optogenetic approaches and best practices continue to emerge, Tremblay suggests that those looking for their own advice read to the end of the Neuron paper too, where members of contributing labs shared some of their own lessons learned. "When I read the advice, I wish I had known everything that people are sharing," he says.

In the meantime, Tremblay is experimenting with a new opsin for manipulating neurons in macaques, one that, per the database, no one has tried just yet. If they had, he'd reach out - to avoid the waste of time and important animals if the protein had proved ineffective elsewhere. He's on his own for now with this particular opsin, but once his results are ready, they'll head online first.

\section{Ellen P. Neff}

Published online: 18 January 2021

https://doi.org/10.1038/s41684-021-00711-4 